\title{
Identifying pre-bariatric subtypes based on temperament traits, emotion dysregulation, and disinhibited eating: A latent profile analysis
}

Running head: Pre-bariatric subtypes

Lisa Schäfer, M.Sc. ${ }^{1 *}$, Claudia Hübner M.Sc. ${ }^{1}$, Thomas Carus, M.D. ${ }^{2}$, Beate Herbig, M.D. ${ }^{3}$, Florian Seyfried, M.D. ${ }^{4}$, Stefan Kaiser, M.D. ${ }^{5}$, Tatjana Schütz, Ph.D. ${ }^{6}$, Arne Dietrich, M.D. ${ }^{1}$, \& Anja Hilbert, Ph.D. ${ }^{1}$

${ }^{1}$ Leipzig University Medical Center, Integrated Research and Treatment Center AdiposityDiseases, Medical Psychology and Medical Sociology, Philipp-Rosenthal-Strasse 27, 04103 Leipzig, Germany, E-mail: claudia.huebner@medizin.uni-leipzig.de, arne.dietrich@medizin.uni-leipzig.de, anja.hilbert@medizin.uni-leipzig.de.

${ }^{2}$ Department of General Surgery, Asklepios Clinic, Suurheid 20, 22559 Hamburg, Germany, E-mail: t.carus@asklepios.com.

${ }^{3}$ Schön Klinik Hamburg Eilbek Bariatric Clinic, Dehnhaide 120, 22081 Hamburg, Germany, E-mail: bherbig@schoen-kliniken.de.

${ }^{4}$ Department of General, Visceral, Vascular and Pediatric Surgery, University Hospital, University of Würzburg, Oberdürrbacher Strasse 6, 97080 Würzburg, Germany, E-mail: seyfried_f@ukw.de.

${ }^{5}$ Department of Visceral, Pediatric and Vascular Surgery, Hospital Konstanz, Luisenstrasse 7, 78464 Konstanz, Germany, E-mail: stefan.kaiser@klinikum-konstanz.de. 
${ }^{6}$ Leipzig University Medical Center, Integrated Research and Treatment Center

AdiposityDiseases, Core Unit Nutrition and Clinical Phenotyping, Philipp-Rosenthal-Strasse

27, 04103 Leipzig, Germany, E-mail: tatjana.schuetz@medizin.uni-leipzig.de.

* Corresponding author. Leipzig University Medical Center, Medical Psychology and

Medical Sociology, Philipp-Rosenthal-Strasse 27, 04103 Leipzig, Germany. Phone: +49 341

97-15364, Fax: +49 341 97-15359, E-mail: lisa.schaefer@medizin.uni-leipzig.de.

Abstract word count: 202

Word count: 4113

Number of figures: 1

Number of tables: 4 


\section{Acknowledgements}

This work was supported by the Federal Ministry of Education and Research (BMBF), Germany, FKZ: 01EO1501.

The authors thank Christine Stroh, M.D., Wolfgang Tigges, M.D., Christian Jurowich, M.D., Kathrin Hohl, Martina Pabst, M.D., Petra Börner, and Yvonne Sockolowsky for their support in data collection at the study centers, and Ricarda Schmidt, Ph.D., for her editing of the manuscript. 
Abstract

2 Objective: The efficacy of bariatric surgery has been proven; however, a subset of patients

3 fails to achieve expected long-term weight loss postoperatively. As differences in surgery

4 outcome may be influenced by heterogeneous psychological profiles in pre-bariatric patients,

5 previous subtyping models differentiated patients based on temperament traits. The objective

6 of the present study was to expand these models by additionally considering emotion

7 dysregulation and disinhibited eating behaviors for subtyping, as these factors were associated

8 with maladaptive eating behaviors and poor post-bariatric weight loss outcome.

9 Methods: Within a prospective multicenter registry, $N=370$ pre-bariatric patients were examined using interview and self-report questionnaires. A latent profile analysis was

11 performed to identify subtypes based on temperament traits, emotion dysregulation, and 12 disinhibited eating behaviors.

13 Results: Five pre-bariatric subtypes were identified with specific profiles regarding self-

14 control, emotion dysregulation, and disinhibited eating behaviors. Subtypes were associated 15 with different levels of eating disorder psychopathology, depression, and quality of life. The expanded model increased variance explanation compared to temperament-based models.

17 Conclusion: By adding emotion dysregulation and disinhibited eating behaviors to previous

18 subtyping models, specific pre-bariatric subtypes emerged with distinct psychological deficit 19 patterns. Future investigations should test the predictive value of these subtypes for post20 bariatric weight loss and health-related outcomes. 


\section{Introduction}

Bariatric surgery is the most effective treatment option for patients with obesity grade III (body mass index, BMI $\left.\geq 40.0 \mathrm{~kg} / \mathrm{m}^{2}\right)$ and grade II $\left(35.0 \mathrm{~kg} / \mathrm{m}^{2} \leq \mathrm{BMI}<40.0 \mathrm{~kg} / \mathrm{m}^{2}\right)$ with obesity-related comorbidities (e.g., type II diabetes, hypertension, sleep apnea), leading to a clinically relevant excess weight loss and decrease of physical and psychological disorders in the long term. ${ }^{1-3}$ Though, $20-30 \%$ of bariatric patients achieve less than $50 \%$ excess weight loss after surgery, ${ }^{4,5}$ which is one of the key indicators for successful post-bariatric weight outcome.

Differences in bariatric weight loss outcome were discussed to be influenced by heterogeneous psychological profiles in pre-bariatric patients regarding general psychopathology (i.e., depression, anxiety), disinhibited eating behaviors, and associated temperament traits, particularly impulsivity ${ }^{6-8}$ Impulsivity is a multifaceted temperament trait, which involves the tendency to act rashly and spontaneously without consideration of consequences (i.e., disinhibition). ${ }^{9}$ Impulsive behavior is closely connected to affective states, either accompanied by highly positive (i.e., extraversion/sensation seeking) or highly negative affect (i.e., neuroticism/negative urgency) ${ }^{9,10}$ For individuals with obesity and especially for bariatric patients, impulsivity is likely to be triggered by food cues and is particularly associated with negative affect. ${ }^{11-14}$ In addition, pre-bariatric patients often present a range of clinical and subclinical pathological eating behaviors, e.g., binge-eating disorder (BED) ${ }^{15}$ and emotional eating, which are characterized by emotion dysregulation, ${ }^{13,16-18}$ defined as deficits in recognizing and managing negative affect.

Latent profile analysis (LPA) is an empirical technique that aims to identify latent subgroups or profiles of individuals on the basis of observed variables (i.e., indicators). ${ }^{19}$ During the last years, LPA has increasingly gained relevance in eating disorder research; for example, for describing different phenotypical profiles in individuals with $\mathrm{BED},{ }^{20}$ bulimia 
1 nervosa, ${ }^{21}$ anorexia nervosa, ${ }^{22,23}$ and other non-normative eating behaviors. ${ }^{24,25}$ The

2 characterization of distinct subtypes in these samples allows to detect patients' specific

3 psychological pattern and may help to develop treatment programs tailored to individuals'

4 particular needs. Indeed, there is first evidence for the predictive value of empirically derived

5 personality subtypes for treatment outcome (e.g., objective binge-eating episodes and purging

6 frequency) in bulimia nervosa. ${ }^{26}$ In pre-bariatric patients, LPA would be valuable for

7 identifying subgroups which are at risk for insufficient surgery outcome. However, a cluster

8 analytic approach has only been applied in two studies so far $^{27,28}$ which subtyped patients

9 based on temperament traits.

Using the Big Five personality traits (i.e., neuroticism, extraversion, openness to

11 experience, agreeableness, and conscientiousness), ${ }^{27}$ two clusters in $N=102$ pre-bariatric

12 women were found by using K-means cluster analysis: a 'resilient/high functional' subtype

13 with a normal personality profile and a 'dysregulated/undercontrolled' subtype. Patients of the

14 latter cluster, characterized by high neuroticism, low extraversion, and low conscientiousness,

15 showed higher negative affect, less cognitive control, and a greater general and eating

16 disorder psychopathology than the 'resilient' subtype. Müller et al. ${ }^{28}$ replicated these two

17 clusters by using latent profile analysis: they investigated temperament traits measured by the

18 Behavioral Inhibition System and Behavioral Activation System (BIS/BAS) scales and the

19 Effortful Control subscale of the Adult Temperament Questionnaire-Short Form (ATQ-EC) in

20 a clinical sample of $N=156$ patients with obesity mostly seeking for bariatric treatment.

21 Patients from the first cluster, referred to as 'emotionally dysregulated/undercontrolled'

22 subtype, showed significantly higher levels of reward and punishment sensitivity and lower

23 levels of effortful control in comparison to the second cluster, referred to as 'resilient/high

24 functional' subtype. Furthermore, the first cluster demonstrated more eating disorder 
1 psychopathology, higher occurrence of BED, greater depression, and a higher probability of

2 adult attention-deficit/hyperactivity disorder than the 'resilient/high functional' subtype.

4 disinhibited eating behaviors for subtyping pre-bariatric patients, although the predictive

5 value of these intrapersonal factors for post-bariatric weight outcome was suggested. ${ }^{29-32}$ This

6 study aimed to provide a fine-grained analysis of pre-bariatric subtypes by additionally taking

7 emotion dysregulation and disinhibited eating behaviors into account. First, the temperament-

8 based model was tested with the expectation to replicate the two previously found subtypes.

9 Second, the temperament-based model was extended by including emotion dysregulation and

10 disinhibited eating behaviors as indicators for clusters. We hypothesized that the extended

11 model would explain more variance compared to the temperament-based model. Ultimate

12 goal of this pre-bariatric subtyping is to help identify patients at high risk for low post-

13 bariatric weight loss and increased need for targeted psychological intervention.

Materials and Methods

\section{Participants}

This study was part of the multicenter Psychosocial Registry for Bariatric Surgery (PRAC) study, which longitudinally assesses psychosocial aspects in a consecutive sample of bariatric surgery patients (for a detailed description, see ${ }^{16}$ ). The study included preoperative data of $N=370$ patients who applied for bariatric surgery in six participating study centers in Germany from March 2012 to August 2016. All patients provided written informed consent before study participation according to procedures approved by the authorized ethics committee of each study center. Data collection proceeded independently of clinical treatment, and all patients were informed that study data would be treated as strictly confidential and inaccessible to the surgical team. 
Measures for Cluster Identification

Reactive temperament - punishment and reward sensitivity. To measure patients' reactive temperament, total scores of the Behavioral Inhibition System (BIS) scale and the Behavioral Activation System (BAS) scale ${ }^{33}$ were used. The BIS scale (Cronbach's $\alpha$ in this study's sample $=.72$ ) measures individual dispositional differences in punishment sensitivity, whereas the BAS scale $(\alpha=.82)$ assesses the dispositional sensitivity to reward. Regulative temperament - effortful control. Regulative temperament was assessed by computing the total score of the effortful control subscale of the Adult Temperament Questionnaire-Short Form (ATQ-EC ${ }^{34} ; \alpha=.77$ ). Effortful control allows performing an act even in the presence of strong avoidance tendencies and regulates the reactive temperament. Emotion dysregulation. The total score of the Difficulties in Emotion Regulation Scale $\left(\mathrm{DERS}^{35} ; \alpha=.94\right)$ was used to assess deficits in recognizing and managing negative affect. Disinhibited eating behaviors. Emotional eating (EE) and eating in the absence of hunger $(\mathrm{EAH})$ were assessed to determine disinhibited eating behaviors in patients. For EE, the total score of the emotional eating subscale of the Dutch Eating Behavior Questionnaire $\left(\mathrm{DEBQ}-\mathrm{EE}^{36} ; \alpha=.95\right.$; German version by Grunert ${ }^{37}$ ) was computed. EAH (i.e., starting or continuing eating due to negative affect, fatigue/boredom, or external cues) was assessed by an adapted version $(\alpha=.88)$ of the Eating in the Absence of Hunger scale $\left(\mathrm{EAH}^{38} ;\right.$ German translation by $\mathrm{AH}$ - unpublished manuscript). Total scores were computed.

Measures for Cluster Validation Eating disorder diagnoses. The semi-structured Eating Disorder Examination

24 interview $(\mathrm{EDE})^{39}$ was applied and its diagnostic items were analyzed in order to derive a diagnosis of BED according to the fifth edition of the Diagnostic and Statistical Manual of 
1 Mental Disorders (DSM-5). ${ }^{15}$ In addition, an EDE module for the night eating syndrome

2 (NES) $)^{16}$ was used to diagnose NES according to DSM-5. ${ }^{15}$ Based on previous

3 recommendations, NES was defined as having at least two excessive evening and/or night

4 eating episodes per week during the last three months. ${ }^{40}$

Eating disorder psychopathology. Patients' concerns and behaviors related to eating restraint, body shape, and weight were measured by the global score of the Eating Disorder

7 Examination-Questionnaire $\left(\mathrm{EDE}_{-} \mathrm{Q}^{41} ; \alpha=.86\right)$.

Food addiction. Addictive eating was measured by the Yale Food Addiction Scale

$9 \quad$ YFAS $\left.^{42} ; \alpha=.90\right)$, which assesses seven food addiction symptoms (e.g., tolerance,

withdrawal). Food addition was diagnosed if patients fulfilled more than two symptoms and

11 reported clinically significant distress.

Depression. The total score of the Patient Health Questionnaire Depression Scale

13 (PHQ- $9^{43} ; \alpha=.85$ ), which covers each of the nine DSM-5 criteria for depression, ${ }^{15}$ was

14 computed and a cut-off of 15 was applied to determine probable cases of depression. ${ }^{44}$

15 Quality of life. The total score of the Impact of Weight on Quality of Life-Lite

16 (IWQoL-Lite ${ }^{45} ; \alpha=.94$ ) was used to assess individual's quality of life regarding physical

17 function, self-esteem, sexual life, public distress, and work.

Weight status. Body Mass Index (BMI, $\mathrm{kg} / \mathrm{m}^{2}$ ) was calculated from measured weight and height for $n=320$ patients $(86.5 \%)$. If measured weight and height were not available, self-reported weight and height were used based on the very high correlation between

21 measured and self-reported BMI in this study's sample $(r=.95)$.

Data Analytic Plan

Analyses comprised three steps: first, the temperament-based model was tested by performing latent profile analysis (LPA) on the basis of reactive (BIS, BAS) and regulative 
1 (ATQ-EC) temperament as indicator variables (Model 1). Second, LPA was repeated by

2 adding three additional indicator variables, including emotion dysregulation (DERS), emotional eating (DEBQ-EE), and eating in the absence of hunger (EAH), to the prior model (Model 2). Third, the expanded model was validated by comparing the identified subtypes with regard to sociodemographic variables, BED and NES diagnosis, eating disorder psychopathology, food addiction, depression diagnosis, and quality of life. LPAs were carried out using Latent Gold Version $4.5{ }^{46}$ Total scores of the indicator variables were available for all patients. LPAs were performed with one to eight clusters and the most parsimonious number of latent classes was determined by examining the Bayesian information criterion (BIC). ${ }^{47}$ BIC was chosen as primary fit index as it is more conservative

11 than the indices based on the Akaike information criterion (AIC), which tend to overestimate the true number of clusters. ${ }^{48}$ Additionally, the Akaike information criterion (AIC), ${ }^{49}$ the

13 Akaike information criterion 3 (AIC3), ${ }^{50}$ and entropy-values were reported. Lowest values of the three information criteria are indicative for the best-fitting model and higher entropy values indicate better classification accuracy. In addition to global measures of model fit, bivariate residuals as local fit indices were examined. High bivariate residuals indicate remaining correlations between two indicators within clusters that were not adequately explained by the model. In this case, the conditional independence assumption is not met. In the present study, direct effects, demonstrated by bivariate residuals larger than $3.84,{ }^{46}$ were added to the model if this procedure resulted in a more parsimonious cluster solution. After

21 determining the number of clusters, participants were assigned to a cluster on the basis of 22 their highest probability. Univariate analyses of variance (ANOVAs), $\chi^{2}$ tests and, in terms of violation of 24 normality and homogeneity of variances, non-parametric tests were used to compare and validate the clusters identified in Model 2. Post-hoc tests with Bonferroni correction were 
1 applied to examine pair-wise differences if omnibus tests were significant. Significant

2 differences in explained variances $R^{2}$ between Model 1 and Model 2 were examined by $z$ tests

3 for correlation coefficients with a two-tailed $\alpha$ of .05 using $z$-transformed $R .^{51}$ All statistical

4 tests, carried out using SPSS Version 23.0 (IBM Corp. Released 2015. IBM SPSS Statistics

5 for Windows, Version 23.0. Armonk, NY: IBM Corp), were two-tailed and considered

6 significant when $p$ values were $<.05$.

Results

8

\section{Latent Profile Analysis: Temperament-based Model (Model 1)}

Fit indices of the LPA models based on temperament traits for the one- to eight-cluster solutions are displayed in Table 1. The BIC was lowest for the two-cluster model, whereas the AIC and AIC3 indicated a three- and four-cluster solution, respectively. The two-cluster solution was chosen as it was selected by BIC and represented the most parsimonious model. The selected model explained $R^{2}=.51$ of variance by cluster membership and had an entropy value of .46 .

The clusters differed significantly in terms of BIS, BAS, and ATQ-EC. Compared to Cluster $2(n=142)$, Cluster $1(n=228)$ showed significant lower levels of BIS, $F(1,368)=$ $164.56, p<.001$, and higher levels of BAS, $F(1,368)=14.82, p<.001$, and ATQ-EC, $F(1$, 
$1368)=342.65, p<.001$. Cluster 1 was labeled as 'Resilient/high functioning' and Cluster 2 as

2 'Emotionally dysregulated/undercontrolled' subtype.

3

\section{Latent Profile Analysis: Extended Model (Model 2)}

The fit indices for the extended LPA models are displayed in Table 2. The BIC suggested a model with five clusters due to BIC and the principle of parsimony. The AIC indicated an eight-cluster solution, whereas the AIC3 indicated a model with seven clusters. The direct effect between the indicators DEBQ-EE and EAH, indicated by the bivariate residual of 13.80, was included. The final model explained $R^{2}=.73$ of variance and had an entropy value of .76. Accordingly, the five-cluster solution of Model 2 explained significantly more variance than the two-cluster solution found in Model 1, $z=5.10, p<.001$.

\section{Characterization of Pre-bariatric Subtypes (Model 2)}

Figure 1 depicts the profile plots of the five detected clusters of Model 2 characterized by their $z$-transformed scores of the six indicator variables. Indicators' total scores and group differences between pre-bariatric subtypes are summarized in Table 3.

Cluster 1 comprised $17.6 \%(n=65)$ of the sample and was characterized by the lowest levels of BIS, DERS, EAH, and DEBQ-EE compared to the other subtypes. Patients from this cluster were labeled as 'Resilient' as they also reported the highest levels of ATQ-EC, together with those from Cluster 5.

Cluster $2(n=109 ; 29.5 \%)$ showed a similar profile as the 'Resilient' subtype, as both clusters did not differ significantly in BIS, BAS, and DERS ( $p s=.358-.808)$. However, patients from this cluster, referred to as 'Slightly reduced control $(R C)$ ' subtype, reported significantly lower levels of ATQ-EC $(p=.026)$ and higher levels of EAH and DEBQ-EE ( $p$ s $<.001)$ compared to the 'Resilient' cluster. 
Cluster $3(n=114 ; 30.8 \%)$, which was labeled as 'Moderately $R C$ ' subtype, was

2 characterized by moderate levels of BIS, DERS, EAH, and DEBQ-EE and showed the second

3 lowest level of all clusters with regard to ATQ-EC values. In addition, this subtype had the

4 lowest BAS scores of all subtypes, however, they did not differ significantly from those found

5 in Cluster $4(p=.289)$ and Cluster $5(p=.073)$.

Cluster 4, referred to as 'Severely $R C$ ' subtype, comprised $18.4 \%(n=68)$ of the sample and showed the highest levels of BIS and DERS and the lowest level of ATQ-EC compared to all other subtypes $(p s<.001)$. Furthermore, patients of this subtype reported the second highest levels of EAH and DEBQ-EE.

Cluster 5 comprised the minority of the sample $(n=14 ; 3.8 \%)$. Patients of this cluster were characterized by low levels of DERS and the highest levels of ATQ-EC. In this regard, total scores of this cluster did not differ significantly from those reported in the 'Resilient' subtype (DERS: $p=.639$; ATQ-EC: $p=.140$ ). However, patients of this cluster showed the same pattern as the 'Severely RC' subtype in regard to EAH $(p=.380)$, and even significantly higher levels of DEBQ-EE $(p=.015)$ and, therefore, were labeled 'Food-specifically $R C$ '. BIS scores of these patients were low, but significant differences solely emerged in comparison to the 'Severely RC' subtype $(p<.001)$, whereas the reported BAS scores of these patients did not differ significantly from those found in all other pre-bariatric subtypes $(p s=.073-.801)$.

\section{Validation of Pre-bariatric Subtypes (Model 2)}

As presented in Table 4, there were no differences among the subtypes with respect to age, BMI, sex, and education ( $p s=.081$ - .664). Descriptively, BED and NES were most prevalent in the 'Severely RC' cluster ( $n=10,17.2 \%$ and $n=9,15.5 \%$, respectively), followed by the 'Moderately' ( $n=3,2.9 \%$ and $n=8,7.8 \%$, respectively) and 'Slightly RC' 
1 subtype ( $n=3,3.0 \%$ and $n=5,5.0 \%$, respectively). No cases of BED and NES were reported

2 in the 'Resilient' and 'Food-specifically RC' subtype. In this regard, both subtypes differed

3 significantly from the 'Severely RC' cluster $(p s=.001-.024)$. Half of the 'Severely RC'

4 patients $(n=36,52.9 \%)$ met criteria for food addiction. High rates of food addiction were

5 also found in the 'Food-specifically RC' $(n=5,35.7 \%)$ subtype, which did not differ

6 significantly in this respect from the 'Severely $\mathrm{RC}^{\prime}(p=.240)$ and 'Moderately $\mathrm{RC}^{\prime}$ group (n

$7=34,30.1 \% ; p=.667)$, whereas food addiction occurred significantly less in the 'Resilient' ( $n$

$8=10,9.3 \%)$ and 'Slightly RC' subtype $(n=4,6.2 \% ; p s=.001-.005)$. Accordingly, highest

9 levels of eating disorder psychopathology were reported by patients from the 'Severely' and

10 'Food-specifically RC' subtype, which did not differ significantly from each other $(p=.480)$,

11 while the 'Resilient' and 'Slightly RC' subtype reported the lowest values compared to the

12 other groups $(p s=.001-.002)$. Depression was most prevalent in the 'Severely RC' cluster $(n$ $13=30,44.1 \%)$, followed by the 'Moderately $\mathrm{RC}^{\prime}(n=14,12.3 \%)$ and 'Food-specifically RC'

14 subtypes $(n=1,7.1 \%)$, while both clusters did not differ significantly from each other in this

15 respect $(p=.573)$. Little to no cases of depression were reported in the Slightly $\mathrm{RC}^{\prime}$ and

16 'Resilient' group, which did not show any significant group differences $(p=.177)$. With

17 regard to quality of life, lowest levels were found in patients of the 'Severely RC' subtype,

18 followed by the 'Food-specifically', 'Moderately', and 'Slightly RC' cluster, whereas the

19 'Resilient' patients significantly reported the highest levels compared to the remaining

20 clusters $(p s=.001-.036)$.

Discussion

Based on a large multicenter sample of pre-bariatric patients, the present study

provides first evidence that temperament traits, emotion dysregulation, and disinhibited eating behaviors are key features in pre-bariatric patients, accounting for almost three quarters of the 
1 heterogeneity in psychological profiles in this sample. Using LPA, five subtypes with distinct

2 psychological profiles were identified and associated with different prevalence rates of eating

3 disorder diagnoses and varying levels of eating and general psychopathology. Based on the

4 high accuracy and gain in explained variance by assigning pre-bariatric patients to one of five

5 psychological profiles, the present investigation showed more favorable results than previous

6 subtyping studies using temperament traits only. ${ }^{27,28}$

Most patients were classified as 'Moderately RC' subtype, characterized by low levels of effortful control and high levels of punishment sensitivity, emotion dysregulation, as well as frequent disinhibited eating, including emotional eating and eating in the absence of hunger. Patients belonging to the 'Severely RC' subtype showed psychological profiles

11 similar to the 'Moderately RC' subtype, although they were even higher in impairment. The 12 profiles of these two subtypes correspond to the 'emotionally dysregulated/undercontrolled' 13 cluster of pre-bariatric patients derived from temperament-based models. ${ }^{27,28}$ Thus, the 14 present study provided a more precise picture of different levels of self-control and emotion regulation in pre-bariatric patients by distinguishing the 'Resilient' and 'Slightly RC' subtypes at the healthier end of the spectrum from the 'Moderately RC', 'Severely RC', and 'Food-specifically RC' subtypes at the more pathological, less controlled end. Most cases of BED, NES, food addiction, and highest levels of eating disorder psychopathology were found among patients of the 'Severely RC' subtype, followed by the 'Moderately' and 'Slightly RC' subtype. This is consistent with previous findings indicating that deficits in self-control and

21 emotion regulation in pre-bariatric patients are closely connected to a range of pathological eating behaviors. ${ }^{16-18,53,54}$ Further, previous studies revealed that pre- and post-bariatric BED, 23 binge eating, loss of control eating, ${ }^{55-57}$ and emotional eating ${ }^{58}$ are negative predictors for post-bariatric weight loss outcome in the long term. Consequently, patients of the 'Severely RC' subtype are expected to be at higher risk for unfavorable weight loss, disturbed eating 
1 behaviors, and psychological impairments after surgery compared to the other subtypes. Thus,

2 longitudinal investigations are required to test the predictive value of the five subtypes in

3 terms of surgery outcome.

In accordance with temperament-based models, ${ }^{27,28}$ 'Resilient' patients did not report

5 deficits in self- and emotion regulation, little to no disinhibited eating, and no cases of BED

6 and NES were found. The prevalence of food addiction in this cluster (6.2\%) was lower than

7 those found in other pre-bariatric samples (14.0-16.5). ${ }^{54,59}$ A small group of patients showed

8 functional levels of self- and emotion regulation, which were comparable with the 'Resilient'

9 group, but high levels of emotional eating and eating in the absence of hunger. This subtype,

10 termed 'Food-specifically RC', did not differ from the 'Severely' and 'Moderately RC'

11 subtypes in terms of eating disorder psychopathology and prevalence rates of food addiction.

12 However, there were no cases of BED and NES in this group suggesting that patients from

13 this cluster might rather show subclinical eating disturbances, such as grazing and snacking,

14 instead of meeting full-syndrome criteria for an eating disorder, although this was not

15 assessed in the current study. As subclinical eating disturbances are highly prevalent in pre-

16 bariatric patients and associated with negative surgery outcome,${ }^{56,60}$ future investigations are

17 warranted to examine whether patients of the 'Food-specifically RC' cluster are at risk for

18 poor weight outcome in the long term.

Regarding general psychopathology, highest rates for probable cases of depression

were found in the 'Severely RC' subtype, followed by the 'Moderately' and 'Food-

21 specifically RC' group. Current depression rates in 'Severely RC' patients (44.1\%) were

22 considerably higher than those found in other pre-bariatric samples (3.4-25.3\%), ${ }^{8}$ indicating

23 that this cluster should receive particular attention of clinicians. Previous research indicated

24 that negative mood was associated with uncontrolled eating in pre-bariatric patients with both

25 impulsive personality and deficits in emotion regulation, ${ }^{12,13}$ suggesting that patients of the 
1 'Severely RC' subtype show problematic eating behaviors in order to regulate their negative

2 feelings as they may lack alternative, healthier strategies. Of note, little to no probable cases

3 of depression were detected in the 'Slightly RC' and 'Resilient' group which is in line with

4 the results on eating disorder diagnoses. Regarding quality of life, subtypes characterized by

5 frequent disinhibited eating and increased eating disorder psychopathology (i.e., 'Severely

6 RC', 'Food-specifically RC', and 'Moderately RC' subtypes) reported poorer quality of life

7 than patients from the 'Resilient' and 'Slightly RC' subtypes, which is in accordance with

8 previous research. ${ }^{61}$

Major strengths of this study include the application of LPA as an empirically driven approach to subtype pre-bariatric patients in a large multicenter sample. Well-established

11 instruments were used to assess eating disorder diagnoses and psychopathology, independent

12 from clinical routines related to surgery. As a limitation, the cross-sectional design of the study does not allow for evaluating the predictive value of the identified subgroups on diverse surgery outcomes (e.g., weight loss, quality of life). In addition, several factors which may have an effect on the analyzed variables were not examined, such as medication intake and somatic comorbidities. Finally, for $n=26$ patients, BMI was calculated by using the selfreported body weight and height. However, correlation analyses of objective and subjective BMI data of $n=164$ patients revealed high agreement between these two types of sources $(r=$ $.95)$, thus preventing from strong measurement biases.

Overall, the present findings highlight that pre-bariatric patients show heterogeneous psychological profiles in terms of temperament traits, emotion regulation, and disinhibited eating behaviors. Future studies are needed to replicate the identified subtypes in other prebariatric samples, including the rather small cluster of 'Food-specifically RC' patients. In addition, more research is needed to longitudinally investigate trajectories of psychological profiles after bariatric surgery, because it has been shown that the majority of bariatric 
1 patients improve significantly in terms of depressive symptoms ${ }^{1,2}$ and disinhibited eating

2 behaviors $^{62}$ already six months after surgery. Further, future studies should compare several

3 cluster solutions on different pre- and post-bariatric time points in order to detect the

4 subtyping model with the highest predictive value for post-bariatric excess weight loss

5 outcome and other psychological aspects (e.g., patients' adherence at follow-up) and,

6 accordingly, determining the optimal assessment point for psychological evaluation to detect

7 and treat high-risk patients as early as possible.

8 Several clinical implications derive from the present findings. Clinicians may be

9 advised to systematically assess temperament, emotion dysregulation, and disinhibited eating

10 behaviors during pre-operative psychological evaluation in order to detect pre-bariatric

11 patients showing profiles of potential vulnerability for post-bariatric maladaptive eating

12 behaviors, insufficient weight loss, and psychosocial impairment. For orientation, clinicians

13 may refer to cut-off scores of instruments assessing deficits in self- and emotion regulation

14 (e.g., ATQ-EC, DERS). In addition, patients showing a psychological profile congruent with

15 the 'Severely RC' and 'Moderately RC' subtypes, i.e., difficulties with self- and emotion

16 regulation as shown by similar mean scores of measures, should receive particular attention

17 from clinicians as these subgroups may be prone for post-bariatric complications. First

18 evidence indicated that cognitive-behavioral interventions in pre-bariatric patients hold

19 promise for reducing eating disorder psychopathology and depressive symptomatology ${ }^{63}$ and

20 that post-bariatric behavioral interventions improve post-surgery weight loss. ${ }^{64,65}$ Although

21 psychological examinations should be standard for all pre-bariatric patients, it remains unclear

22 which patients are in need for additional psychosocial interventions to improve post-bariatric

23 diet compliance, weight loss, and quality of life. 
Conflict of Interest Statement

The authors declare no conflicts of interest with respect to the content of this manuscript.

Statement of Human and Animal Rights

All procedures performed in studies involving human participants were in accordance with the ethical standards of the institutional research committees and with the 1964 Helsinki declaration and its later amendments or comparable ethical standards.

Statement of Informed Consent

Informed consent was obtained from all individual participants included in the study. 


\section{References}

1. Burgmer, R., Legenbauer, T., Müller, A., de Zwaan, M., Fischer, C., \& Herpertz, S. (2014). Psychological outcome 4 years after restrictive bariatric surgery. Obes Surg, 24, 1670-1678. doi: 10.1007/s11695-014-1226-x

2. de Zwaan, M., Enderle J., Wagner S., Mühlhans, B., Ditzen, B., Gefeller O., ... Müller, A. (2011). Anxiety and depression in bariatric surgery patients: a prospective, follow-up study using structured clinical interviews. J Affect Disord, 133, 61-68. doi: 10.1016/j.jad.2011.03.025

3. Puzziferri, N., Roshek, T. B., Mayo, H. G., Gallagher, R., Belle S. H., \& Livingston, E. H. (2014). Long-term follow-up after bariatric surgery: a systematic review. JAMA, 312, 934-942. doi: 10.1001/jama.2014.10706

4. Balsiger, B. M., Murr, M. M., Poggio, J. L., \& Sarr, M. G. (2000). Bariatric surgery. Surgery for weight control in patients with morbid obesity. Med Clin North Am, 84, 477-489. doi: 10.1016/S0025-7125(05)70232-7

5. Courcoulas, A. P., Christian, N. J., Belle, S. H., Berk, P. D., Flum, D. R., Garcia, L., ... Wolfe, B. M. (2013). Weight change and health outcomes at 3 years after bariatric surgery among individuals with severe obesity. JAMA, 310, 2416-2425. doi: 10.1001/jama.2013.280928

6. Eldar, S., Heneghan, H. M., Brethauer, S. A., \& Schauer, P. R. (2011). Bariatric surgery for treatment of obesity. Int J Obes (Lond), 35, S16-S21. doi: 10.1038/ijo.2011.142

7. Livhits, M., Mercado, C., Yermilov, I., Parikh, J. A., Durson, E., Mehran, A., ... Gibbons, M. M. (2012). Preoperative predictors of weight loss following bariatric surgery: systematic review. Obes Surg, 22, 70-89. doi: 10.1007/s11695-011-0472-4 
8. Malik, S., Mitchell, J. E., Engel, S., Crosby, R., \& Wonderlich, S. (2014).

Psychopathology in bariatric surgery candidates: a review of studies using structured diagnostic interviews. Compr Psychiatry, 55, 248-259. doi:

10.1016/j.comppsych.2013.08.021

9. Sharma, L., Markon, K. E., \& Clark, L. A. (2014). Toward a theory of distinct types of "impulsive" behaviors: a meta-analysis of self-report and behavioral measures. Psychol Bull, 140, 374-408. doi: 10.1037/a0034418

10. Gullo, M. J., Loxton, N. J., \& Dawe, S. (2014). Impulsivity: four ways five factors are not basic to addiction. Addict Behav, 39, 1547-1556. doi:

10.1016/j.addbeh.2014.01.002

11. Gerlach, G., Herpertz, S., \& Loeber, S. (2015) Personality traits and obesity: a systematic review. Obes Rev, 16, 32-63. doi: 10.1111/obr.12415

12. Leehr, E. J., Krohmer, K., Schag, K., Dresler, T., Zipfel, S., \& Giel, K. E. (2015). Emotion regulation model in binge eating disorder and obesity - a systematic review. Neurosci Biobehav Rev, 49, 125-134. doi: 10.1016/j.neubiorev.2014.12.008

13. Schag, K., Mack, I., Giel, K. E., Ölschläger, S., Skoda, E. M., von Feilitzsch, M., ... Teufel, M. (2016). The impact of impulsivity on weight loss four years after bariatric surgery. Nutrients, 8,721 . doi:10.3390/nu8110721

14. Schag, K., Schönleber, J., Teufel, M., Zipfel, S., \& Giel, K. E. (2013). Food-related impulsivity in obesity and binge eating disorder - a systematic review. Obes Rev, 14, 477-495. doi: 10.1111/obr.12017

15. American Psychiatric Association. (2013). Diagnostic and Statistical Manual of Mental Disorders ( $5^{\text {th }}$ ed.). Arlington, VA: American Psychiatric Publishing.

16. Baldofski, S., Tigges, W., Herbig, B., Jurowich, C., Kaiser, S., Stroh, C., ... Hilbert, A. 
(2015). Non-normative eating behavior and psychopathology in prebariatric patients with binge-eating disorder and night eating syndrome. Surg Obes Relat Dis, 11, 621626. doi: 10.1016/j.soard.2014.09.018

17. Koball, A. M., Himes, S. M., Sim, L., Clark, M. M., Collazo-Clavell, M. L., Mundi, M., ... Grothe, K. B. (2016). Distress tolerance and psychological comorbidity in patients seeking bariatric surgery. Obes Surg, 26, 1559-1564. doi: 10.1007/s11695015-1926-X

18. Micanti, F., Iasevoli, F., Cucciniello, C., Costabile, R., Loiarro, G., Pecoraro, G., ... Galletta, D. (2017). The relationship between emotional regulation and eating behaviour: a multidimensional analysis of obesity psychopathology. Eat Weight Disord, 22, 105-115. doi: 10.1007/s40519-016-0275-7

19. Lazerfeld, P. F., Henry, N.W. (1968). Latent structure analysis. Boston, MA: Houghton Mifflin.

20. Peterson, C. B., Crosby, R. D., Wonderlich, S. A., Mitchell, J. E., Crow, S. J., \& Engel, S. (2013). Predicting group cognitive-behavioral therapy outcome of binge eating disorder using empirical classification. Behav Res Ther, 51, 526-532. doi: 10.1016/j.brat.2013.05.001

21. Wonderlich, S. A., Crosby, R. D., Engel, S. G., Mitchell, J. E., Smyth, J., \& Miltenberger, R. (2007). Personality-based clusters in bulimia nervosa: differences in clinical variables and ecological momentary assessment. J Pers Disord, 21, 340-357. doi: 10.1521/pedi.2007.21.3.340

22. Lavender, J. M., Wonderlich, S. A., Crosby, R. D., Engel, S. G., Mitchell, J. E., Crow, S. J. , .. Le Grange, D. (2013). Personality-based subtypes of anorexia nervosa: examining validity and utility using baseline clinical variables and ecological momentary assessment. Behav Res Ther, 51, 512-517. doi: 10.1016/j.brat.2013.05.007 
23. Goldschmidt, A. B., Wonderlich, S. A., Crosby, R. D., Chao, L., Engel, S. G., Lavender, J. M., ... Le Grange, D. (2014). Latent profile analysis of eating episodes in anorexia nervosa. J Psychiatr Res, 53, 193-199. doi: 10.1016/j.jpsychires.2014.02.019

24. Tharner, A., Jansen, P. W., Kiefte-de Jong, J., Moll, H. A., van der Ende, J., Jaddoe, V. W. V., ... Franco, O. H. (2014). Toward an operative diagnosis of fussy/picky eating: a latent profile approach in a population-based cohort. Int J Behav Nutr Phys Act, 11, 14. doi: 10.1186/1479-5868-11-14

25. Vannucci, A., Tanofsky-Kraff, M. Crosby, R. D., Ranzenhofer, L. M., Shomaker, L. B., Field, S. E., ... Yanovski, J. A. (2013). Latent profile analysis to determine the typology of disinhibited eating behaviors in children and adolescents. J Consult Clin Psychol, 81, 494-507. doi: 10.1037/a0031209

26. Haynos, A. F., Pearson, C. M., Utzinger, L. M., Wonderlich, S. A., Crosby, R. D., Mitchell, J. E., ... Peterson, C. B. (2017). Empirically derived personality subtyping for predicting clinical symptoms and treatment response in bulimia nervosa. Int J Eat Disord, 50, 506-514. doi: 10.1002/eat.22622

27. Claes, L., Vandereycken, W., Vandeputte, A., \& Braet, C. (2013). Personality subtypes in female pre-bariatric obese patients: do they differ in eating disorder symptoms, psychological complaints and coping behaviour? Eur Eat Disord Rev, 21, 72-77. doi: 10.1002/erv.2188

28. Müller, A., Claes, L., Wilderjans, T. F., \& de Zwaan, M. (2014). Temperament subtypes in treatment seeking obese individuals: a latent profile analysis. Eur Eat Disord Rev, 22, 260-266. doi: 10.1002/erv.2294

29. Amundsen, T., Strømmen, M., \& Martins, C. (2017). Suboptimal weight loss and weight regain after gastric bypass surgery - postoperative status of energy intake, 
eating behavior, physical activity, and psychometrics. Obes Surg, 27, 1316-1323. doi: $10.1007 / \mathrm{s} 11695-016-2475-7$

30. Mack, I., Ölschläger, S., Sauer, H., von Feilitzsch, M., Weimer, K., Junne, F., ... Teufel, M. (2016). Does laparoscopic sleeve gastrectomy improve depression, stress and eating behaviour? A 4-year follow-up study. Obes Surg, 12, 2967-2973. doi:10.1007/s11695-016-2219-8

31. Marek, R. J., Ben-Porath, Y. S., Dulmen, M. H. , Ashton, K., \& Heinberg, L. J. (2017). Using the presurgical psychological evaluation to predict 5-year weight loss outcomes in bariatric surgery patients. Surg Obes Relat Dis, 13, 514-521. doi: 10.1016/j.soard.2016.11.008

32. Wedin, S., Madan, A., Correll, J., Crowley, N., Malcolm, R., Karl Byrne, T., \& Borckardt, J. J. (2014). Emotional eating, marital status and history of physical abuse predict 2-year weight loss in weight loss surgery patients. Eat Behav, 15, 619-624. doi: 10.1016/j.eatbeh.2014.08.019

33. Carver, C. S. \& White, T. L. (1994). Behavioral inhibition, behavioral activation, and affective responses to impending reward and punishment: the BIS/BAS scales. $J$ Pers Soc Psychol, 67, 319-333. doi:10.1037/0022-3514.67.2.319

34. Derryberry, D. \& Rothbart, M. K. (1988). Arousal, affect, and attention as components of temperament. J Pers Soc Psychol, 55, 958-966. doi: 10.1037//0022-3514.55.6.958

35. Gratz, K. \& Roemer, L. (2004). Multidimensional assessment of emotion regulation and dysregulation: development, factor structure, and initial validation of the Difficulties in Emotion Regulation Scale. J Psychopathol Beh Assess, 26, 41-54. doi: 0.1023/B:JOBA.0000007455.08539.94

36. Van Strien, T., Frijters, J. E. R., Bergers, G. P. A., \& Defares, P. B. (1986). The Dutch Eating Behavior Questionnaire (DEBQ) for assessment of restrained, emotional, and 
external eating behavior. Int J Eat Disord, 5, 295-315. doi: 10.1002/1098108X(198602)5:2<295::AID-EAT2260050209>3.0.CO;2-T

37. Grunert, S. C. (1989). Ein Inventar zur Erfassung von Selbstaussagen zum Ernährungsverhalten [An inventory of the assessment of self-reported eating behavior]. Diagnostica, 35, 167-179.

38. Tanofsky-Kraff, M., Ranzenhofer, L. M., Yanovski, S. Z., Schvey, N. A., Faith, M., Gustafson, J., \& Yanovski, J. A. (2008). Psychometric properties of a new questionnaire to assess eating in the absence of hunger in children and adolescents. Appetite, 51, 148-155. doi: 10.1016/j.appet.2008.01.001

39. Fairburn C. G., Cooper, Z., \& O’Connor, M. (2014). Eating Disorder Examination (17.0D). New York: Guilford Press.

40. Allison, K. C., Lundgren, J. D., O'Reardon, J. P., Geliebter, A., Gluck, M. E., Vinai, P., ... Stunkard, A. J. (2010). Proposed diagnostic criteria for night eating syndrome. Int J Eat Disord, 43, 241-247. doi: 10.1002/eat.20693

41. Fairburn, C. G. \& Beglin, S. J. (2008). Eating Disorder Examination Questionnaire (6.0). In C. G. Fairburn (Ed.), Cognitive Behavior Therapy and Eating Disorders (pp. 309-314). New York: Guilford Press.

42. Gearhardt, A. N., Corbin, W. R., \& Brownell, K. D. (2009). Preliminary validation of the Yale Food Addiction Scale. Appetite, 52, 430-436. doi: 10.1016/j.appet.2008.12.003

43. Spitzer, R. L., Kroenke, K., \& Williams, J. B. (1999). Validation and utility of a selfreport version of PRIME-MD: the PHQ primary care study. JAMA, 282, 1737-1744. doi:10.1001/jama.282.18.1737

44. Cassin, S., Sockalingam, S., Hawa, R., Wnuk, S., Royal, S., Taube-Schiff, M., \& Okrainec, A. (2013). Psychometric properties of the Patient Health Questionnaire 
(PHQ-9) as a depression screening tool for bariatric surgery candidates.

Psychosomatics, 54, 352-358. doi: 10.1016/j.psym.2012.08.010

45. Kolotkin, R. L. \& Crosby, R. D. (2002). Psychometric evaluation of the Impact of Weight on Quality of Life-Lite questionnaire (IWQOL-LITE) in a community sample. Qual Life Res, 11, 157-171. doi:10.1023/A:1015081805439

46. Vermount, J. K. \& Madison, J. (2005). Latent GOLD 4.0 User's Guide. Belmont: Statistical Innovations Inc.

47. Schwarz, G. (1978). Estimating the dimension of a model. Ann Stat, 6, 461-464. doi:10.1214/aos/1176344136

48. Bulteel, K., Wilderjans, T. F., Tuerlinckx, F., \& Ceulemans, E. (2013). CHull as an alternative to AIC and BIC in the context of mixtures of factor analyzers. Behav Res Methods, 45, 782-791. doi: 10.3758/s13428-012-0293-y

49. Akaike, H. (1974). A new look at the statistical model identification. IEEE Trans Automat Contr, 19, 716-723. doi: 10.1109/TAC.1974.1100705

50. Bozdogan, H. (1993). Choosing the number of component clusters in the mixturemodel using a new informational complexity criterion of the inverse-fisher information matrix. In: O. Opitz, B. Lausen, \& R. Klar (Eds.), Information and classification, concepts, methods and applications (pp. 40-54). Berlin: Springer.

51. Howell, D. C. (2011). Statistical Methods for Psychology. Boston: Cengage Learning Emea.

52. Cohen J. (1988). Statistical Power Analysis for the Behavioral Sciences. (2nd ed.). Hillsdale, New Jersey: Erlbaum.

53. Claes, L. \& Müller, A. (2015). Temperament and personality in bariatric surgery resisting temptations? Eur Eat Disord Rev, 23, 435-441. doi: 10.1002/erv.2398 
54. Koball, A. M., Clark, M. M., Collazo-Clavell, M., Kellogg, T., Ames, G., Ebbert, J., \& Grothe, K. B. (2016). The relationship among food addiction, negative mood, and eating-disordered behaviors in patients seeking to have bariatric surgery. Surg Obes Relat Dis, 12, 165-170. doi: 10.1016/j.soard.2015.04.009

55. Chao, A. M., Wadden, T. A., Faulconbridge, L. F., Sarwer, D. B., Webb, V. L., Shaw, J. A., ... Williams, N. N. (2016). Binge-eating disorder and the outcome of bariatric surgery in a prospective, observational study: two-year results. Obesity (Silver Spring), 24, 2327-2333. doi: 10.1002/oby.216

56. Conceição, E. M., Utzinger L. M., \& Pisetsky, E. M. (2015). Eating disorders and problematic eating behaviours before and after bariatric surgery: characterization, assessment and association with treatment outcomes. Eur Eat Disord Rev, 23, $417-$ 425. doi: 10.1002/erv.2397

57. Wimmelmann, C. L., Dela, F., \& Mortensen, E. L. (2014). Psychosocial predictors of weight loss after bariatric surgery: a review of the recent research. Obes Res Clin Pract, 8, e299-e313. doi: 10.1016/j.orcp.2013.09.003

58. Castellini, G., Godini, L., Amedei, S. G., Faravelli, C., Lucchese, M., \& Ricca, V. (2014). Psychosocial effects and outcome predictors of three bariatric surgery interventions: a 1-year follow-up study. Eat Weight Disord, 19, 217-224. doi: $10.1007 / \mathrm{s} 40519-014-0123-6$

59. Brunault, P., Ducluzeau P. H., Bourbao-Tournois C., Delbachian, I., Couet, C., Réveillère, C., \& Ballon, N. (2016). Food addiction in bariatric surgery candidates: prevalence and risk factors. Obes Surg, 26, 1650-1653. doi: 10.1007/s11695-0162189-X 
60. Opolski, M., Chur-Hansen, A., \& Wittert, G. (2015). The eating-related behaviours, disorders and expectations of candidates for bariatric surgery. Clin Obes, 5, 165-197. doi: $10.1111 /$ cob.12104

61. Jenkins, P. E., Hoste, R. R., Meyer, C., \& Blissett, J. M. (2010). Eating disorders and quality of life: a review of the literature. Clin Psychol Rev, 31, 113-121. doi: 10.1016/j.cpr.2010.08.003

62. Meany, G., Conceicao, E., \& Mitchell, J. E. (2014). Binge eating, binge eating disorder and loss of control eating: effects on weight outcomes after bariatric surgery. Eur Eat Disord Rev, 14, 87-91. doi: 10.1002/erv.2273

63. Cassin, S., Sockalingam, S., Du, C., Wnuk, S., Hawa, R., \& Parikh, S. V. (2016). A pilot randomized controlled trail of telephone-based cognitive behavioural therapy for preoperative bariatric surgery patients. Behav Res Ther, 80, 17-22. doi:

10.1016/j.brat.2016.03.001

64. Kalarchian, M. A. \& Marcus, M. D. (2015). Psychosocial interventions pre and post bariatric surgery. Eur Eat Disord Rev, 23, 457-462. doi: 10.1002/erv.2392

65. Rudolph, A. \& Hilbert, A. (2013). Post-operative behavioural management in bariatric surgery: a systematic review and meta-analysis of randomized controlled trials. Obes Rev, 14, 292-302. doi: 10.1111/obr.12013 


\section{Table 1}

Fit indices for temperament-based latent profile analyses (Model 1)

\begin{tabular}{|c|c|c|c|c|c|c|}
\hline $\begin{array}{l}\text { No. of } \\
\text { clusters }\end{array}$ & BIC & AIC & AIC3 & LL & Entropy & Largest bivariate residual \\
\hline 1 & 7369.00 & 7345.52 & 7351.52 & -3666.76 & 1.00 & 43.06 \\
\hline 2 & 7357.09 & 7306.21 & 7319.21 & -3640.11 & 0.46 & 3.54 \\
\hline 3 & 7371.12 & 7292.85 & 7312.85 & -3626.42 & 0.57 & 2.03 \\
\hline 4 & 7391.87 & 7286.21 & 7313.21 & -3616.10 & 0.61 & 1.54 \\
\hline 5 & 7421.82 & 7288.76 & 7322.76 & -3610.38 & 0.62 & 1.60 \\
\hline 6 & 7454.35 & 7293.89 & 7334.89 & -3605.95 & 0.61 & 0.84 \\
\hline 7 & 7482.85 & 7295.00 & 7343.00 & -3599.50 & 0.66 & 0.86 \\
\hline 8 & 7511.95 & 7296.71 & 7351.71 & -3593.36 & 0.64 & 0.72 \\
\hline
\end{tabular}

Notes. Best-fitting models are depicted in bold. Higher entropy values indicate better classification accuracy. The largest bivariate residual checks for the conditional independence assumption. $\mathrm{BIC}=$ Bayesian information criterion; $\mathrm{AIC}=$ Akaike information criterion; AIC $3=\mathrm{Akaike}$ information criterion 3; LL = Log-Likelihood. 


\section{Table 2}

Fit indices for extended latent profile analyses (Model 2)

\begin{tabular}{|c|c|c|c|c|c|c|}
\hline $\begin{array}{l}\text { No. of } \\
\text { clusters }\end{array}$ & BIC & AIC & AIC3 & LL & Entropy & Largest bivariate residual \\
\hline 1 & 15865.98 & 15819.02 & 15831.02 & -7897.51 & 1.00 & 250.87 \\
\hline 2 & 15315.07 & 15217.24 & 15242.24 & -7583.62 & 0.85 & 86.80 \\
\hline 3 & 15189.78 & 15051.07 & 15079.07 & -7482.53 & 0.84 & 32.57 \\
\hline 4 & 15182.10 & 14982.51 & 15033.52 & -7440.25 & 0.79 & 19.09 \\
\hline 5 & 15181.77 & 14931.31 & 14995.31 & -7401.65 & 0.82 & 13.80 \\
\hline 6 & 15200.41 & 14899.07 & 14976.07 & -7372.54 & 0.82 & 6.77 \\
\hline 7 & 15235.97 & 14883.75 & 14973.75 & -7351.88 & 0.83 & 5.03 \\
\hline 8 & 15275.16 & 14872.07 & 14975.07 & -7333.04 & 0.85 & 3.71 \\
\hline
\end{tabular}

Notes. Best-fitting models are depicted in bold. Higher entropy values indicate better classification accuracy. The largest bivariate residual checks for the conditional independence assumption. $\mathrm{BIC}=$ Bayesian information criterion; AIC = Akaike information criterion; AIC $3=$ Akaike information criterion 3; LL = Log-Likelihood. 


\section{Table 3}

Characterization of pre-bariatric subtypes with regard to indicator variables (Model 2)

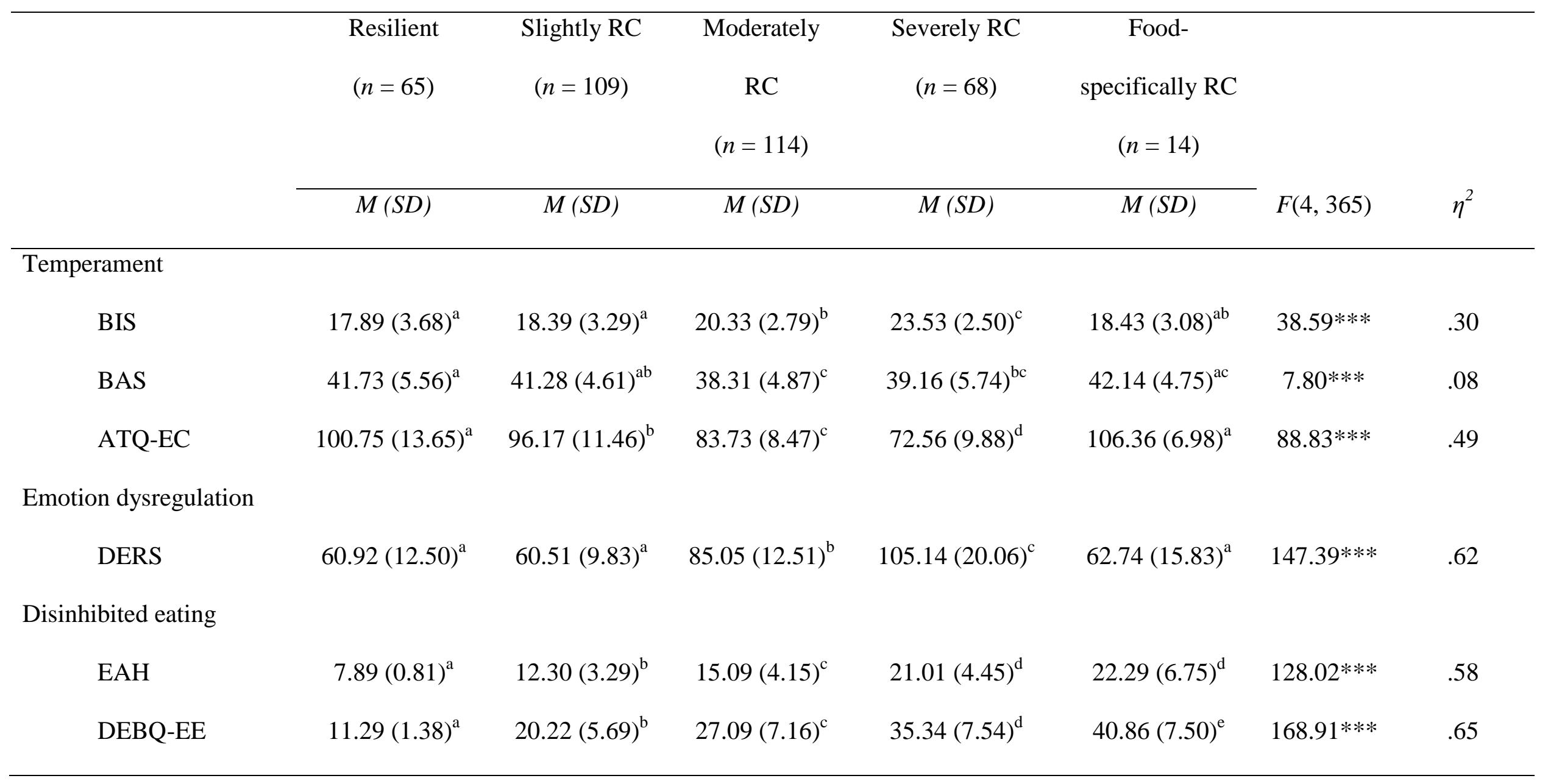


Notes. Superscripts that differ display significant differences between subtypes after post-hoc comparisons with Bonferroni corrections. Effect size $\eta^{2}$ was interpreted according to Cohen (small effect: $.01 \leq \eta^{2}<.06$; medium: $.06 \leq \eta^{2}<.14$; large: $\eta^{2} \geq .14$ ) ${ }^{52}$ RC $=$ reduced control; BIS $=$

Behavioral Inhibition System (7-28*, less favorable scores are asterisked); BAS = Behavioral Activation System (13-52*), ATQ-EC = Effortful Control of the Adult Temperament Questionnaire (19*-133); DERS = Difficulties in Emotion Regulation Scale (36-180*); EAH = Eating in the Absence of Hunger (10-50*); DEBQ-EE = Emotional Eating of the Dutch Eating Behavior Questionnaire (7-35*).

$* * * p<.001$ 


\section{Table 4}

Validation of pre-bariatric subtypes (Model 2)

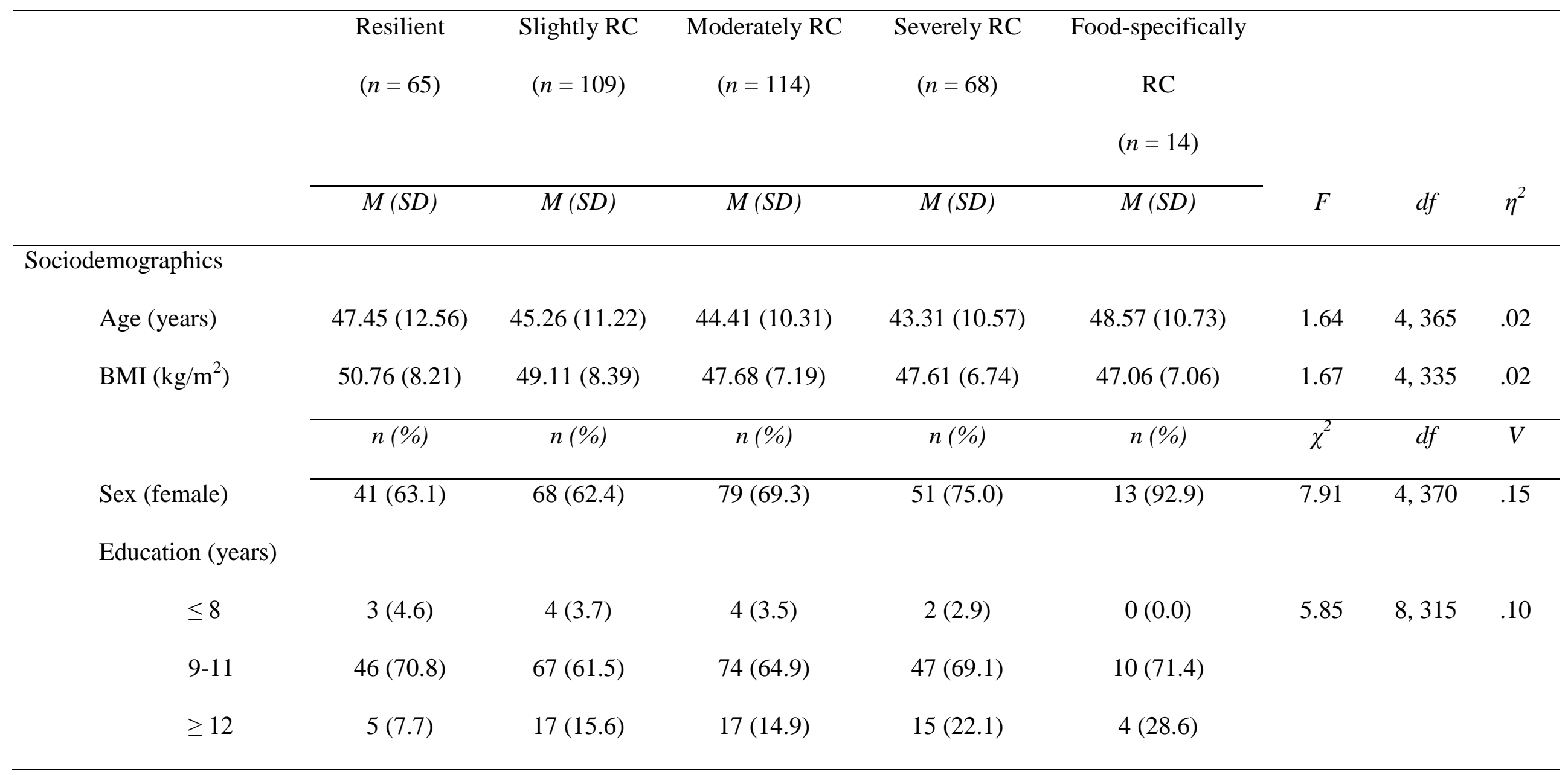


Table 4 continued.

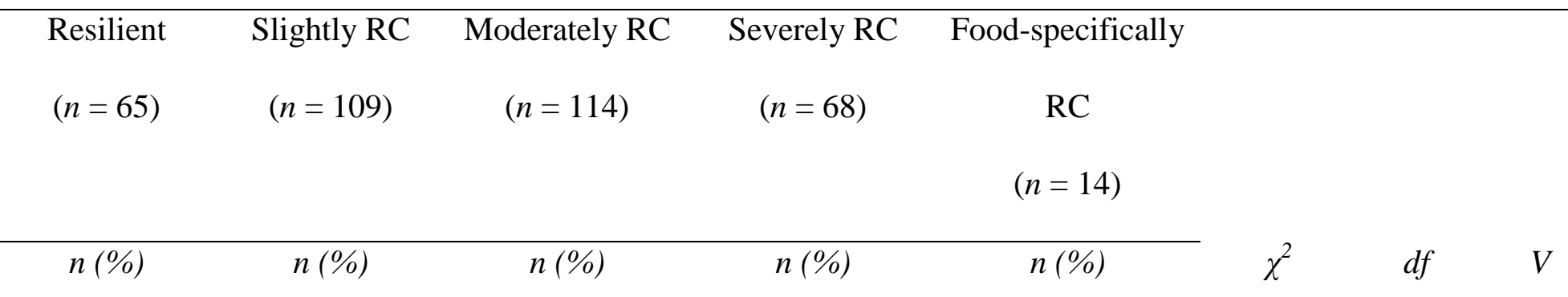

Eating disorder diagnoses

\begin{tabular}{|c|c|c|c|c|c|c|c|}
\hline BED & $0(0.0)^{\mathrm{a}}$ & $3(3.0)^{\mathrm{a}}$ & $3(2.9)^{\mathrm{a}}$ & $10(17.2)^{b}$ & $0(0.0)^{\mathrm{ab}}$ & $25.25 * * *$ & 4,337 \\
\hline JES & $0(0.0)^{\mathrm{a}}$ & $5(5.0)^{\mathrm{ab}}$ & $8(7.8)^{\mathrm{bc}}$ & $9(15.5)^{c}$ & $0(0.0)^{\mathrm{abc}}$ & $13.69 * *$ & 4,337 \\
\hline
\end{tabular}

Food addiction

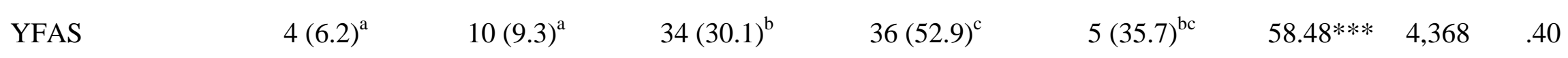

Depression

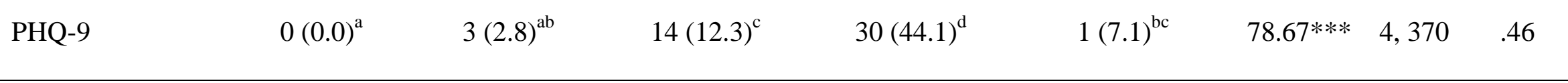


Table 4 continued.

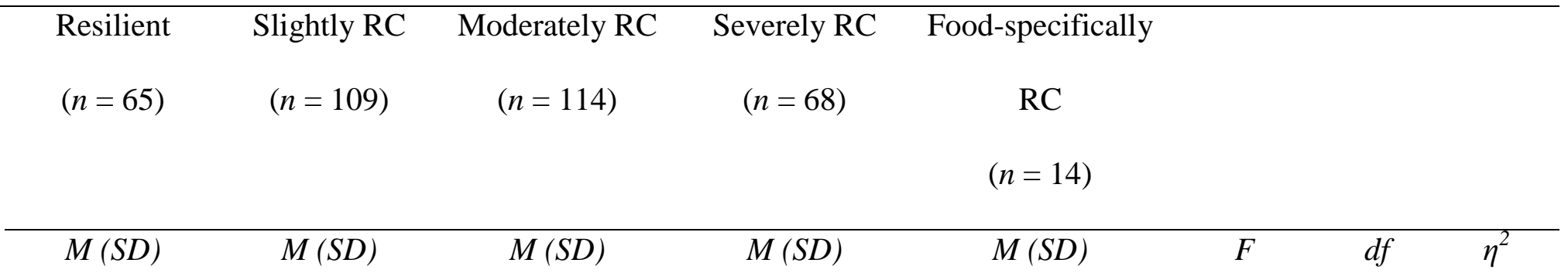

Eating disorder

psychopathology

$\begin{array}{llllllll}\text { EDE-Q global } \quad 2.48(0.90)^{\mathrm{a}} & 2.72(0.80)^{\mathrm{a}} & 3.10(0.90)^{\mathrm{b}} & 3.65(0.97)^{\mathrm{c}} & 3.45(1.00)^{\mathrm{bc}} & 18.59 * * * & 4,365 & .17\end{array}$

Quality of life

$$
\begin{array}{lllllllll}
\text { IWQoL-Lite } \quad 83.65(25.28)^{\mathrm{a}} & 91.92(24.80)^{\mathrm{b}} & 102.63(21.90)^{\mathrm{c}} & 118.12(16.66)^{\mathrm{d}} & 103.14(23.38)^{\mathrm{bcd}} & 23.08 * * * & 4,364 & .20
\end{array}
$$

Notes. Superscripts that differ display significant differences between subtypes after post-hoc comparisons with Bonferroni corrections. Effect size $\eta^{2}$ and Cramer's $V$ were interpreted according to Cohen (small effect: $.01 \leq \eta^{2}<.06$, medium: .06 $\leq \eta^{2}<.14$, large: $\eta^{2} \geq .14$; small effect: .10 $\leq V<$ .30 , medium: $.30 \leq V<.50$, large: $V \geq .50) .{ }^{52} \mathrm{RC}=$ reduced control; BED = binge-eating disorder; NES = night eating syndrome; YFAS = Yale Food Addiction Scale; PHQ-9 = Public Health Questionnaire-Depression; EDE-Q = Eating Disorder Examination-Questionnaire $(0-6 *$, less favorable scores are asterisked); IWQoL-Lite = Impact of Weight on Quality of Life-Lite (31-155*).

$* * * p<.001, * * p<.01$ 
Figure legend

Figure 1. Profile plots of the pre-bariatric subtypes.

Notes. The figure depicts the standardized scores of the six indicator variables for each of the five clusters of the extended latent profile analysis. $\mathrm{RC}=$ reduced control; $\mathrm{BIS}=$ Behavioral Inhibition System; BAS = Behavioral Activation System, ATQ-EC $=$ Effortful Control of the Adult Temperament Questionnaire; DERS = Difficulties in Emotion Regulation Scale; EAH $=$ Eating in the Absence of Hunger, DEBQ-EE = Emotional Eating of the Dutch Eating Behavior Questionnaire. 


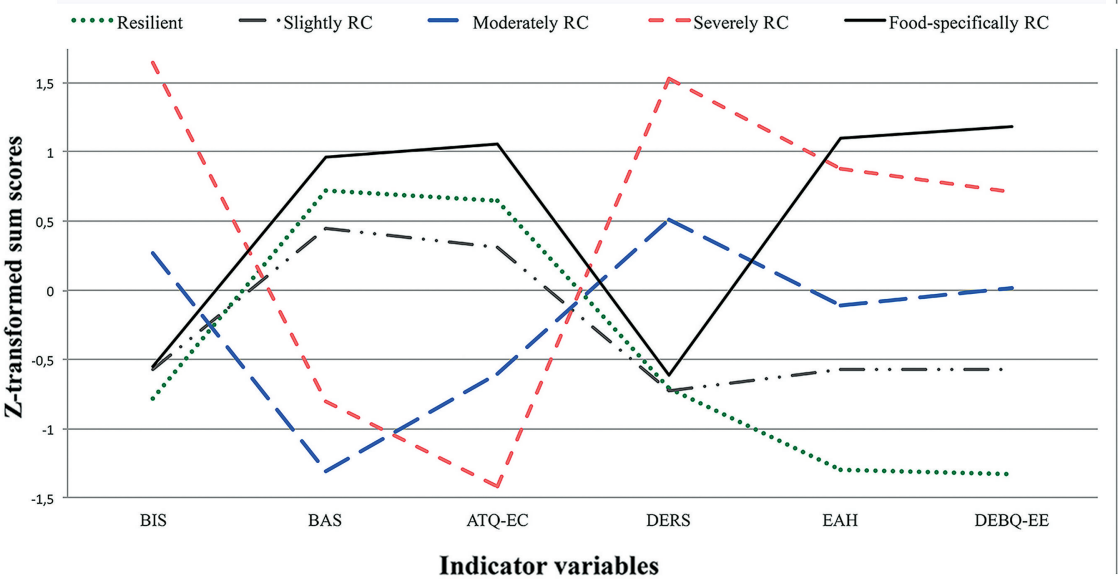

